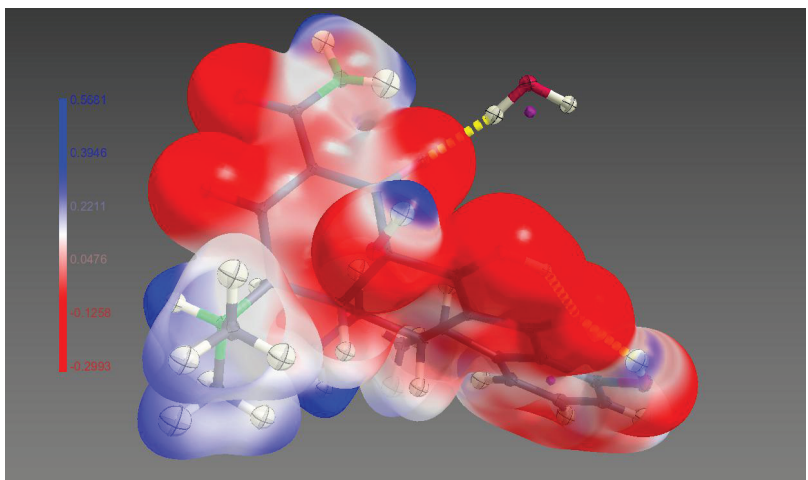

Figure 1. The electrostatic potential around doxycycline moiety in doxycycline monohydrate.

Keywords: doxycycline, antibiotic, protein-ligand interactions

\section{MS28-P3 Dithiadiazolyl radicals - structures and charge densities of their crystals and cocrystals}

Sławomir Domagała ${ }^{1}$, Krzysztof Kość $^{1}$, Sean W. Robinson ${ }^{2}$, Delia A. Haynes ${ }^{2}$, Krzysztof Woźniak ${ }^{1}$

1. Department of Chemistry, University of Warsaw, Warszawa, Poland

2. Department of Chemistry and Polymer Science, Stellenbosch University, Republic of South Africa

email: slawdom@chem.uw.edu.pl

Purely organic systems can exhibit conductivity, superconductivity or magnetically ordered phases properties usually thought as restricted only for crystals containing metallic centres $(\mathrm{Cu}, \mathrm{Mn}$, etc.). One of the most intriguing groups of such systems is a family of dithiadiazolyl radicals.[1] These radicals are chemically stable, so they can be arranged in closely packed structures. Relatively high electrostatic polarization allows for inter- and intramolecular S...N interactions. In the crystal phase, the dithiadiazolyl group often co-exists with a phenyl molecular fragment, which further stabilizes the crystal lattice by introducing an intermolecular $\pi . . . \pi$ aromatic interactions (e.g. phenyl...perfluorophenyl stack interaction). The spin structure of these compounds is strongly coupled to the crystal structure. One can then try to adjust the magnetic properties (e.g. FM-AFM ordering) of such systems by small changes of structural parameters (e.g. distances between molecules in stack). This and other intriguing properties suggest that the dithiadiazolyl radicals are promising candidates for the construction of molecular devices.

The scope of the work was to determine the quantitative electron density distribution and its parameters ( $\rho$ and $\Delta \rho$ in critical points, integrated charges, etc.) for the series of model crystals of radicals belonging to dithiadiazolyl family.[2] The Hansen-Coppens multipole expansion of electron density model was refined against the high resolution $\left(\sin \theta / \lambda>0.7 \AA^{-1}\right)$. X-ray diffraction data to obtain the best models of the electron density distribution in given crystals. These models were then used to calculate quantitative electron density properties using the Bader's Quantum Theory of Atoms in Molecules (QTAIM) such as critical points parameters $\left(\rho_{\mathrm{CP}}, \Delta \rho_{\mathrm{CP}}\right.$, bond paths), atomic basins or integrated electron density parameters (integrated charges, atomic multipoles and volumes, etc). The obtained results and detailed analysis of dithiadiazolyl radicals should hopefully help in a better understanding of the magnetic phenomena in organic systems.

[1] Rawson, J. M., Alberola, A., Whalley, A., J. Mater. Chem. 16, (2006), 2560-2575. [2] C. Allen, D.A. Haynes, C.M. Pask, J.M. Rawson, CrystEngComm 11, (2009), 2048-2050.

Keywords: electron charge density, radicals, cocrystals 